\title{
Synovial Sarcoma in Head and Neck: A Case Report
}

\author{
Adriano Santana Fonseca ${ }^{1}$ Amanda Canário Andrade Azevedo ${ }^{1} \quad$ Fabíola Moreira Magalhães ${ }^{1}$ \\ Nilvano Alves de Andrade ${ }^{1}$ \\ ${ }^{1}$ Santa Casa de Misericórdia da Bahia, Salvador, BA, Brazil \\ Address for correspondence Adriano Santana Fonseca, MD, Santa Casa \\ de Misericórdia da Bahia, Hospital Santa Izabel, Unidade de \\ Int Arch Otorhinolaryngol 2014;18:87-89. \\ Otorrinolaringologia, Praça Conselheiro Almeida Couto, 500, Nazaré, \\ Salvador, Bahia, Brazil 40050-410 (e-mail: adrianootorrino@gmail.com).
}

\begin{abstract}
Introduction Synovial sarcoma is a malignant tumor of mesenchymal pluripotent cells.

Objectives We present a case of synovial sarcoma in the posterolateral wall of the oropharynx.

Resumed report The patient, a 23-year-old woman, was admitted with a history of dysphagia and difficulty in breathing for 8 months, resulting in progressive deterioration and onset of snoring, muffled voice, and local pain. An oropharyngeal tumor in the left posterolateral wall touched the base of the ipsilateral tongue. The patient underwent endoscopic pharyngectomy to remove the lesion. Pathologic examination revealed synovial sarcoma with positive margins, and Mohs technique was proposed for margin control. The margins were disease-free, without the need for total laryngectomy. The pharynx was reconstructed with a microvascular forearm flap. The patient developed postoperative stability.

Conclusion Despite its name, synovial sarcoma is rarely sourced directly from synovial membranes. It is most commonly found in the vicinity of large joints. The location at the head and neck, a location poor in synovial tissue, is unusual. Synovial sarcoma in the head and neck has an aggressive nature and poor prognosis. Resection with negative

Keywords

- sarcoma

- synovial

- deglutition disorders margins remains the foundation of therapy, which is not so easily achieved in the head and neck. It is important for the otorhinolaryngologist and head and neck surgeon to be familiar with this aggressive tumor, which carries high mortality and morbidity. The appropriate diagnosis and treatment can improve prognosis and patient survival.
\end{abstract}

\section{Introduction}

Synovial sarcoma is a malignant tumor of pluripotent mesenchymal cells, ${ }^{1-4}$ most commonly found in periarticular areas, with few cases occurring in the head and neck. ${ }^{1-5}$ The first synovial sarcoma was described by Jernstrom in $1954 .{ }^{6}$ In this article, we present a case of synovial sarcoma in the posterolateral wall of the oropharynx. This case report was authorized by the ethics committee by the number 229.577.

received

June 26, 2013

accepted

September 10, 2013
DOI http://dx.doi.org/

10.1055/s-0033-1361081. ISSN 1809-9777.

\section{Case Report}

The patient, a 23-year-old woman, was admitted to the otorhinolaryngology clinic at our hospital with a history of dysphagia and difficulty of breathing for 8 months, resulting in progressive worsening and emergence of snoring, muffled voice, and local pain. She denied fever episodes, smoking, and alcohol consumption. Physical examination revealed an oropharyngeal tumor in the left posterolateral wall near the base of the tongue. Rhinoscopy and otoscopy confirmed the

Copyright $@ 2014$ by Thieme Publicações License terms Ltda, Rio de Janeiro, Brazil

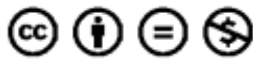




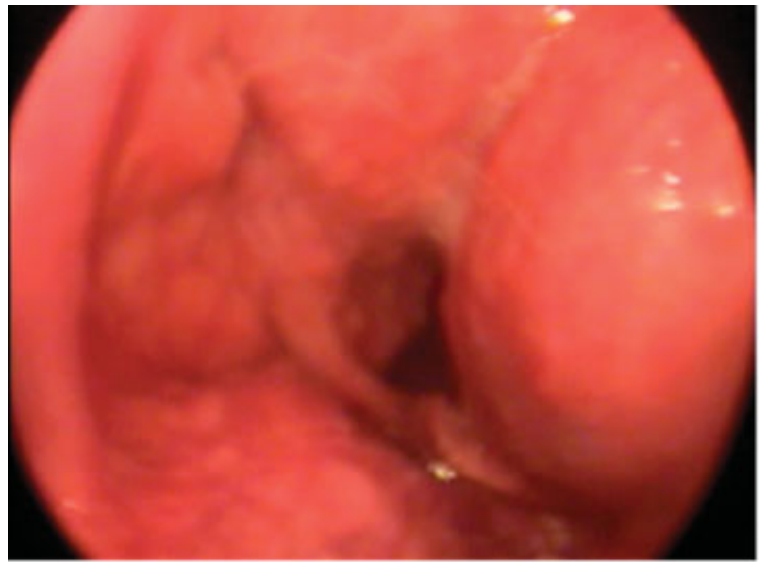

Fig. 1 Preoperative laryngoscopy showing the mass at the hypopharynx.

diagnosis, with no changes. Videolaryngoscopy (-Fig. 1) showed a rounded submucosal tumor with intact mucosa, starting at the left posterolateral wall, touching the epiglottis down to the left, narrowing the light of the entire pharynx, preventing full view of the glottis. Cervical magnetic resonance imaging (- Figs. 2, 3, and $\mathbf{4}$ ) showed a solid cystic lesion in the submucosal left posterolateral wall of the oropharynx of relatively delimited contour, reducing the airway and rejecting the left piriform sinus. The mass measured $\sim 5.3 \times 3.9 \times 2.2 \mathrm{~cm}$ in its greatest axis. Its left lateral region was predominately solid, showing heterogeneous impregnation of paramagnetic contrast, and measured $2.1 \times 2.0 \mathrm{~cm}$, with the cleavage plane delimited by prevertebral muscles. Lymph nodes measured up to $1.3 \mathrm{~cm}$ in internal high jugular chains and submandibular (IB and IIA) on the right. Laboratory tests were unchanged.

Endoscopic pharyngectomy was used to excise the tumor. Pathologic examination revealed a synovial sarcoma with positive margins, and Mohs technique was proposed for

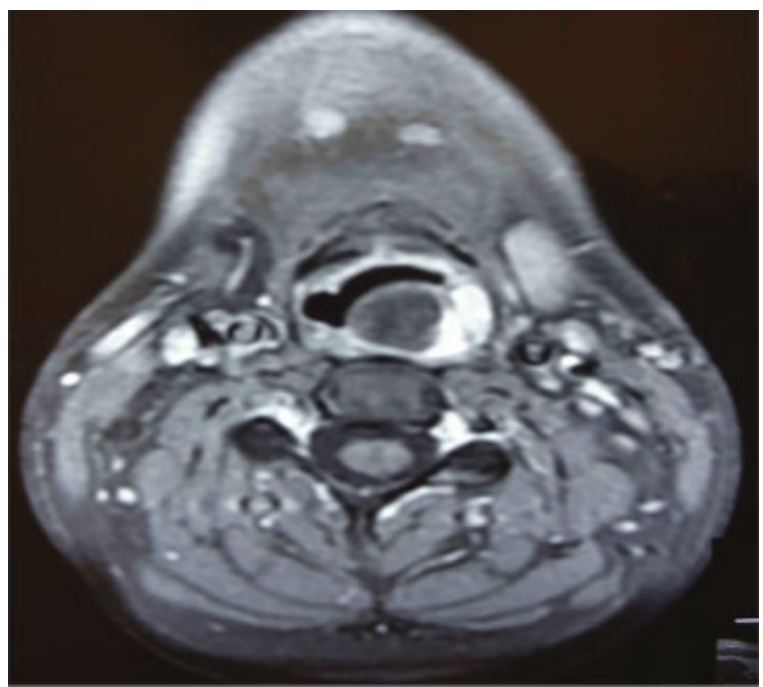

Fig. 2 Nuclear magnetic resonance, axial view.

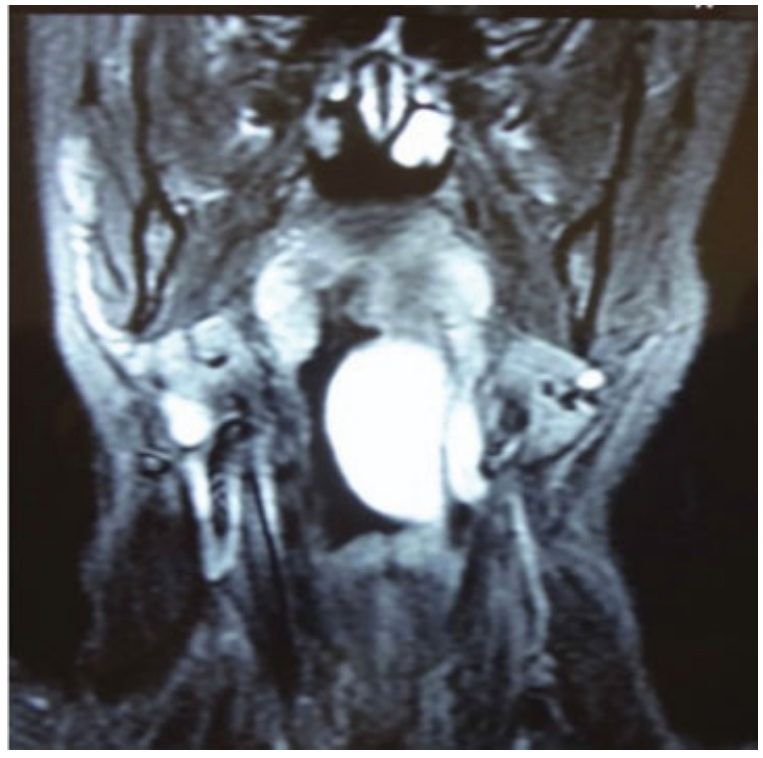

Fig. 3 Nuclear magnetic resonance, coronal view.

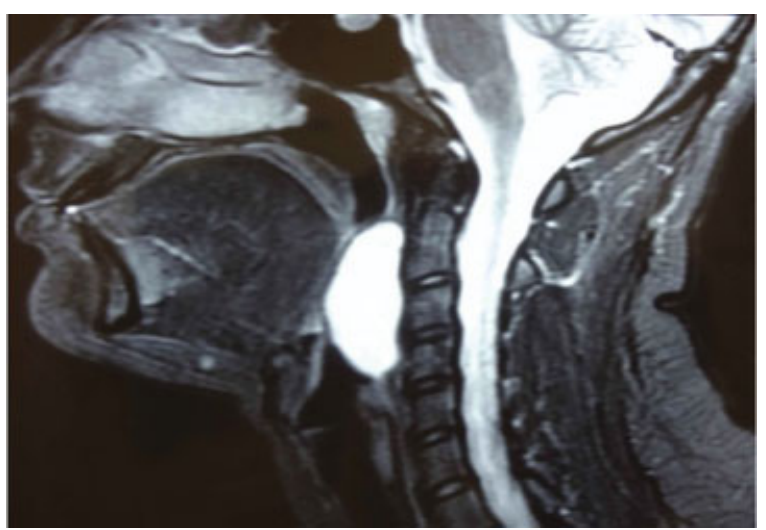

Fig. 4 Nuclear magnetic resonance, sagittal view.

edge control. A full pharyngectomy with ipsilateral modified radical neck dissection was proposed, with the possibility of full laryngeal pharyngectomy depending on surgical margins obtained intraoperatively by freezing anatomy. During surgery, the margins came initially committed, and after expansion, the margins were disease-free. It was possible to obtain free surgical margins without the need for total laryngectomy. The pharynx was reconstructed with a microvascularized forearm flap. We also performed the proposed neck dissection, tracheostomy, and passage of a nasogastric tube for nutrition. The neck dissection was disease-free.

Postoperatively, the patient's condition was stable. Upon follow-up with an oncologist, chemotherapy and radiation were given. A gastrostomy was performed for nutrition, as oral feeding was impossible. At swallowing endoscopy, there was saliva aspiration due to lack of mobility of the pharyngeal muscles. The patient is already in speech therapy. All mobility of the larynx was preserved. Within $\sim 1$ year postoperatively, the patient continues with locoregional control without recurrence and performs pharyngoesophageal dilatations to 
treat actinic stenosis. Return to oral feeding is in progress. The tracheostomy tube is closed and will be removed as soon as oral feeding is stabilized.

\section{Discussion}

Despite its name, synovial sarcoma rarely originates in the synovial membranes. ${ }^{7}$ It is most commonly found in the vicinity of large joints. Occurrence in the head and neck, a location poor in synovial tissue, is uncommon. ${ }^{8}$ Synovial sarcoma comprises 8 to $10 \%$ of all soft tissue sarcoma, ${ }^{1,3,5,7,9}$ and it is estimated that $\sim 3$ to $10 \%$ of sarcomas occur in the head and neck. ${ }^{4,5,10}$ These tumors can be located in the prevertebral space from the base of the skull to the hypopharynx, retropharyngeal and parapharyngeal spaces, and anterior neck along the edges of the sternocleidomastoid muscle, as well as sites in the oropharynx and larynx. ${ }^{8,10}$ Synovial sarcomas show a male predominance (3:2) and predilection for patients between 25 and 36 years of age. ${ }^{4,11,12}$

This type of tumor usually appears as an asymptomatic mass until it acquires sufficient volume to cause compressive effects on neighboring structures. ${ }^{1}$ It can be a painless mass or associated with symptoms such as pain, earache, sore throat, and bleeding. There are two subtypes: monophasic and biphasic. For diagnosis, it is important to include laryngoscopy and head, neck, and chest computed tomography to define the local extent of disease and metastasis. ${ }^{4}$

Resection with negative margins remains the foundation of therapy, ${ }^{7,12}$ which is not so easy to achieve in the head and neck. ${ }^{4,5}$ Local excision is followed by high recurrence rates (60 to $90 \%$ ), usually within 2 years. ${ }^{4,5,9}$ Metastasis to regional lymph nodes of synovial sarcomas in the head and neck is not seen often, although there can be clinically enlarged lymph nodes. ${ }^{5}$ Most metastasis originate from hematogenous dissemination, although up to $20 \%$ spread through the lymphatics to regional lymph nodes. ${ }^{4,8}$ Cervical lymph node dissection is not routinely performed, except in the presence of enlarged lymph nodes. ${ }^{8,10}$ Hematogenous dissemination is the harbinger of death. Pre- and postoperative radiotherapy, with or without chemotherapy, may be responsible for the increase in survival statistics over the last 2 to 3 decades, but studies are still controversial and inconclusive., , 7,9 $^{-1}$

Despite relatively slow growth, long-term survival for synovial sarcomas from anywhere, including the head and neck, is not good. ${ }^{5}$ Synovial sarcoma in the head and neck has an aggressive nature and a reserved prognosis. ${ }^{3,8}$ Several factors have been evaluated for prognosis, and the most significant were tumor size and deep extension at the time of primary treatment, ${ }^{9}$ with survival varying inversely with these two factors. ${ }^{5}$ The age at diagnosis seems to have involvement with prognosis, ${ }^{9}$ although the relative youth of patients with synovial sarcoma of the head and neck does not seem to give a significantly better prognosis. ${ }^{5}$

\section{Final Comments}

Synovial sarcoma is a rare malignant tumor which treatment is essentially surgical resection with high rates of relapse. Although traditional treatment for hypopharynx tumors has been laryngectomy, it can be modified for sarcomas at this area. In these cases, most important are the border's microscopic control and lack of lymphatic metastasis. Radiation therapy with or without chemotherapy appears to be responsible for the increased survival in recent decades. It is important for the otolaryngologist and head and neck surgeon to be familiar with this aggressive tumor, which carries high mortality and morbidity. The appropriate early diagnosis and treatment can improve the prognosis and survival of patients.

\section{References}

1 Saydam L, Kizilay A, Kalcioglu MT, Mizrak B, Bulut F. Synovial sarcoma of the pharynx: a case report. Ear Nose Throat J 2002; 81(1):36-39

2 Tillich M, Ranner G, Humer-Fuchs U, Lang-Loidolt D. Synovial sarcoma of the parapharyngeal space: CT and MRI. Neuroradiology 1998;40(4):261-263

3 Ramamurthy L, Nassar WY, Hasleton PS, Gattamaneni HR, Orton CI. Synovial sarcoma of the pharynx.J Laryngol Otol 1995;109(12): 1207-1210

4 Balakrishnan V, Flatman S, Dixon BJ, Lyons B. Synovial sarcoma of the pharynx causing airway obstruction. Med J Aust 2012;196(1):72-73

5 Carrillo R, Rodriguez-Peralto JL, Batsakis JG. Synovial sarcomas of the head and neck. Ann Otol Rhinol Laryngol 1992;101(4): 367-370

6 Jernstrom P. Synovial sarcoma of the pharynx; report of a case. Am J Clin Pathol 1954;24(8):957-961

7 Betal D, Babu R, Mehmet V. Monophasic synovial sarcoma of the pharynx: a case report. Int Semin Surg Oncol 2009;6:9

8 Bukachevsky RP, Pincus RL, Shechtman FG, Sarti E, Chodosh P. Synovial sarcoma of the head and neck. Head Neck 1992;14(1):44-48

9 Italiano A, Penel N, Robin YM, et al. Neo/adjuvant chemotherapy does not improve outcome in resected primary synovial sarcoma: a study of the French Sarcoma Group. Ann Oncol 2009;20(3):425-430

10 Shaariyah MM, Mazita A, Masaany M, Razif MY, Isa MR, Asma A. Synovial sarcoma: a rare presentation of parapharyngeal mass. Chin J Cancer 2010;29(6):631-633

11 Moore DM, Berke GS. Synovial sarcoma of the head and neck. Arch Otolaryngol Head Neck Surg 1987;113(3):311-313

12 Lee N, Shin E. Treatment outcomes for patients with synovial sarcoma of the head and neck. Expert Rev Anticancer Ther 2008; $8(3): 371-373$ 